\title{
VALIDACIÓN DEL HÍBRIDO DE MAÍZ (Zea mayz L.) DE CRUZA DOBLE H-512 EN EL ESTADO DE VERACRUZ, MÉXICO1
}

\author{
Oscar Tosquy ${ }^{2}$, Mauro Sierra ${ }^{2}$, Flavio Rodríguez $z^{2}$, Ramón Castillo ${ }^{2}$, Joaquín Ortíz ${ }^{3}$, \\ Carlos Tinoco ${ }^{2}$, Alfredo Sandoval ${ }^{2}$, Sergio Uribe ${ }^{2}$
}

\begin{abstract}
RESUMEN
Validación del híbrido de maíz (Zea mayz L.) de cruza doble H-512 en el estado de Veracruz, México. Los resultados sobresalientes del programa de investigación de maíz con respecto a la formación de genotipos más eficientes en el uso de los recursos disponibles, así como el desarrollo de la tecnología necesaria para su aplicación, exigen establecer parcelas semicomerciales donde se prueba a una escala mayor la relevancia de dichos resultados y se verifique la bondad de tal modalidad bajo las condiciones de clima, suelo y manejo por parte de los agricultores. El objetivo de este trabajo fue validar y demostrar el comportamiento del híbrido de maíz H-512 en la región tropical del estado de Veracruz. Por lo anterior se establecieron parcelas de validación durante el ciclo primavera verano de 1992 en las localidades de Cotaxtla, Acayucan, San Andrés Tuxtla y Papantla, Ver. Se incluyeron los genotipos H512, HCSV-23, D-471 x TTC-63, D-471 x ST-549 y los testigos VS-536, B-830 y C-343. El área de las parcelas fue de 2 ha, es decir, cada genotipo fue sembrado en 0,25 ha y se aplicó el paquete tecnológico sugerido para cada localidad. Se encontró que en las localidades de Cotaxtla y Acayucan, el híbrido H-512 registró los mejores rendimientos con 7605 y 6438 kg/ha respectivamente. En San Andrés Tuxtla y Soledad de Doblado, este híbrido fue superado por el híbrido experimental de cruza simple HCSV-23. En promedio de las cinco localidades el H512 presentó los más altos rendimientos y registró una altura de planta intermedia, buena cobertura de mazorca, buena sanidad de planta y mazorca y resistente al acame. Podemos concluir que el H-512 se adapta a las áreas tropicales del estado de Veracruz.
\end{abstract}

\begin{abstract}
Validation of the H-512 double-cross coro (Zea mayz L.) hybrid in the state of Veracruz-México. The outstanding results of the corn research program, with respect to the production of more efficient genotypes in the use of the available resources, as well as the development of the technology needed for its application, call for the establishment of semi-commercial plots, where such results can be tried and its kindness verified under the climate, soil and the farmers' management. The objective of this trial was to validate and show the performance of the H-512 corn hybrid on the tropical region of the State of Veracruz. Validation plots were established during the Spring-summer of 1992 at the localities of Cotaxtla, Acayucan, San Andres Tuxtla and Papantla, Yeracruz. The H-512, HCSV-23, D-471 x TTC-63, D-471 x ST549 and the controls VS-536, 8-830 and C-343 genotypes were included. The plots' area was 2 hectares each, thus every genotype was planted on 0.25 ha and the cultural practices applied were the ones suggested for each zone. The H-512 hybrid registered the highest yields in Cotaxtla and Acayucan with 7605 and $6438 \mathrm{~kg} / \mathrm{ha}$, respectively. This hybrid was surpassed by the simple-cross experimental hybrid HCSV-23 in San Andres Tuxtla and Soledad de Doblado. As an average of the five localities, the H-512 showed the highest yields and registered an intermediate plant height, good husk cover, good plant and ear health and lodging resistant. It can be concluded that the H-512 hybrid adapts well to the tropical zones of Veracruz.
\end{abstract}

\section{INTRODUCCIÓN}

El maíz humilde es el cultivo de los campesinos y alimento único de las masas mexicanas, forma la base de uno de los más grandes mercados agropecuarios con una producción de 12 millones de toneladas obtenidas de 7 millones de ha de siembra. En el trópico húmedo de México se obtiene el $40 \%$ de la producción nacional de maíz,

\footnotetext{
1 Presentado en la XL Reunión Anual del PCCMCAen Costa Rica, América Central. 13 al 19 de marzo, 1994.

2 Ing. Agr. Investigadores del Programa de Maíz en el CIRGOC. INIFAP. SARH. Apdo. Postal 429. Veracruz, Ver.

$3 \mathrm{PhD}$. Asesor del Programa de Maíz en Veracruz.
} 
en esta región, hay aproximadamente un millón de hectáreas de buen temporal, con un rendimiento promedio de 2 toneladas por hectárea, también existen cerca de 100 mil hectáreas de riego, donde la media de rendimiento es de 3 toneladas por hectárea siendo el potencial de rendimiento para esta área de $8 \mathrm{t} / \mathrm{ha}$. Sin embargo uno de los principales problemas que se tienen es el desconocimiento por parte de los productores de la tecnología desarrollada por la investigación.

El uso de semilla mejorada es un elemento estratégico y clave para lograr incrementos en la productividad de maíz y la elección de una buena semilla es el primer paso para aprovechar de forma óptima los recursos disponibles. Para lo cual se validó el híbrido de cruza doble H512 teniendo como testigos la variedad sintética VS-536 y V -530 del INIF AP y los híbridos B-830 y C-343 de las compañías Dekalb y Cargill respectivamente.

El programa de maíz del Campo Experimental Cotaxtla desde sus inicios ha considerado la importancia del establecimiento de parcelas de validación de genotipos, prácticas agronómicas, producción de semillas etc., con agricultores cooperantes, escuelas de enseñanza técnica y dependencia del sector agropecuario donde se prueba a una escala mayor los resultados experimentales.

Durante el temporal de 1991 en la región central de Veracruz, se establecieron parcelas de validación de genotipos de maíz en los municipios de Medellín de Bravo, Tlalixcoyan, Veracruz y Cotaxtla, donde se incluyeron los híbridos de cruza doble HCDV-8, HCDV-9, HCDV-10 (H512) experimentales y H-509 (T), los híbridos de cruza simple experimentales HCSV-28 y HCSV-29 y las variables VS-536, Sint. 6, Sint.9 y V-530. Los resultados indicaron que el HCDV -10 (H-512) con rendimiento de 5550 $\mathrm{kg} / \mathrm{ha}$ superó en $9 \%$ al testigo comercial H-509.

En relación con las variables sintéticas, la VS-536 registró los más altos rendimientos y representa la mejor opción para las siembras en ambientes que no reporten deficiencias de humedad.
La transferencia de tecnología es un tema de gran consideración en el ámbito de investigación agrícola, en este sentido se ha demostrado que la adopción de la tecnología se facilita considerablemente cuando se logra vincular la participación directa entre productores, asistencia técnica e investigación. Calva (1990).

El proceso de transferir tecnología es complejo, más aún cuando el usuario tiene limitados recursos y no puede correr riesgos aceptando innovaciones tecnológicas para él desconocidas; por lo que se hace necesario involucrarlo dentro del proceso de generación y validación de la tecnología a transferir. Ralda (1979).

Palafox (1990), menciona que los resultados de investigación, la validación de tecnología generada y las parcelas de demostración efectuadas por el INIFAP, han demostrado que existe tecnología con un potencial superior a la tecnología que actualmente manejan los productores de diversos cultivos, especialmente en torno al cultivo de maíz.

El mismo autor reporta estudios de adopción indicando que los bajos rendimientos se deben al manejo de tecnología tradicional poco productiva, lo cual es el resultado de un arraigo de la misma, falta de información y capacitación sobre las nuevas técnicas generadas por la investigación.

Sierra et al (1993), menciona que el híbrido H-512 registró una media ponderada de $6052 \mathrm{~kg} / \mathrm{ha}$ a través de 19 ambientes durante 1987 a 1990 superando en rendimiento a los híbridos H-507 y H-511 en un 15,22 y 20\% respectivamente. Así como también presentó menor altura de planta y como consecuencia un porcentaje más bajo de plantas acamadas.

El mismo autor resalta algunas ventajas relativas con respecto a la producción de semilla, ya que sus progenitores no presentan problema en su mantenimiento, sincronía de floración y producción de polen. 
Palafox et al (1991), reporta en una evaluación de genotipos con tecnología PRONAMA Ten la localidad de Tlalixcoyan Veracruz en el ciclo de PV-1990 que el HCDV-10 (H-512 obtuvo el mayor rendimiento con 5168 $\mathrm{kg} / \mathrm{ha}$ en comparación de los testigos comerciales CP561, H-509 y A-681 con 3153, 3036 y 2958 kg/ha respectivamente.

El objetivo es validar y demostrar el comportamiento del híbrido de maíz H-512 en la región tropical del estado de Veracruz.

\section{MATERIALES Y METODOS}

\section{Localización}

Para este estudio se establecieron parcelas demostrativas en las principales áreas maiceras del estado de Veracruz en las localidades de: Campo Experimental Cotaxtla y Plan de Oros en la zona centro del estado, Colonia Agrícola Michapan y Río Tuxtla en la zona sur y Agua Dulce en la zona norte de Veracruz.

El Cuadro 1 muestra localidad y tipo de clima, donde se establecieron las parcelas demostrativas.

Cuadro 1. Localidades de las parcelas demostrativas de maíz en el estado de Veracruz. 1992 B.

\begin{tabular}{lll}
\hline Localidad & \multicolumn{1}{c}{ Municipio } & Clima \\
\hline CECOT & Medellín de Bravo & $\mathrm{Aw}_{1}$ \\
Plan de Oros & Adalberto Tejeda & $\mathrm{Awo}$ \\
Colonia Agrícola & Acayucan & $\mathrm{Am}$ \\
Michapan & & \\
Río Tuxtla & San Andrés Tuxtla & $\mathrm{Aw}_{2}$ \\
Agua Dulce & Papantla & $\mathrm{Aw}_{2}$ \\
\hline
\end{tabular}

\section{Material genético}

El germoplasma utilizado en las parcelas demostrativas durante el ciclo de temporal de 1992 son los siguientes:

$\begin{array}{ll}\text { GENOTIPO } & \text { COMPAÑIA } \\ \text { H-512 } & \text { INIFAP } \\ \text { HCSV-23 } & \text { INIFAP } \\ \text { D-471 X TTC-63 } & \text { INIFAP } \\ \text { D-471 X ST-549 } & \text { INIFAP } \\ \text { B-830 } & \text { DEKALB } \\ \text { C-343 } & \text { CARGILL } \\ \text { VS-536 } & \text { INIFAP } \\ \text { V-530 } & \text { INIFAP }\end{array}$

El área de la parcela fue de 2,0 ha, ya que cada genotipo tuvo una superficie de 0,25 ha. La siembra se efectuó. de acuerdo al paquete tecnológico de maíz sugerido para cada localidad.

Variables y registro de datos:

1. Rendimiento

2. Días a fIor masculina

3. Días a flor femenina

4. Altura de planta

5. Altura de mazorca

6. Acame de raíz

7. Porcentaje de mala cobertura

8. Porcentaje de mazorcas podridas.

Para la estimación del rendimiento se realizaron muestreos, registrándose superficie cosechada, número de plantas y mazorcas cosechadas, porcentaje de grano y de materia seca. Los rendimientos se estimaron al $12 \%$ de humedad; las demás variables fueron tomadas de una muestra de 50 plantas por genotipo.

\section{RESULTADOS Y DISCUSIÓN}

En el Cuadro 2 se observan los resultados a través de localidades en donde el rendimiento mas alto se obtuvo con el híbrido H-512 con 5754 kg/ha, superando sólo en $2 \%$ al promedio de los Testigos Regionales Comerciales (TRC) que rindieron un promedio de $5655 \mathrm{~kg} / \mathrm{ha}$. 
Cuadro 2. Rendimiento promedio ( $\mathrm{kg} / \mathrm{ha}$ ) y características agronómicas de híbridos y variedades en cinco localidades de demostración en el estado de Veracruz y porcentaje de diferenciación con respecto al promedio de los testigos comerciales. Ciclo P.V. 92/92.

\begin{tabular}{lcccccc}
\hline Genotipo & $\begin{array}{c}\text { Rendimiento } \\
\mathbf{X}^{1 /}\end{array}$ & Flor & $\begin{array}{c}\text { Altura } \\
\text { Planta }(\mathbf{c m})\end{array}$ & $\begin{array}{c}\text { Mala } \\
\text { Cobertura }\end{array}$ & \multicolumn{2}{c}{ Porciento } \\
\cline { 6 - 7 } & 5754 & 53 & 247 & 4 & 3 & MZ. Pod. \\
H-512 & 5655 & 53 & 233 & 4 & 1 & Acame \\
TRC & 5421 & 52 & 238 & 9 & 2 & 6 \\
VS-536 & 5235 & 52 & 254 & 6 & 1 & 3 \\
HCSV-23 & 4560 & 49 & 217 & 9 & 0 & 4 \\
V-530 & 4370 & 51 & 214 & 23 & 5 & 8 \\
D-471 X TTC-63 & 3869 & 53 & 213 & 5 & 1 & 5 \\
D-471 X ST-549 & 386 & &
\end{tabular}

" Rendimiento promedio de las localidades: Cotaxtla, Plan de Oros, Agua Dulce, Michapan y Río Tuxtla.

Estos híbridos se comportaron como de ciclo intermedio de 49 a 53 días a flor masculina, siendo el genotipo B-830 de Dekalb el híbrido relativamente mas tardío y el que presentó los más bajos porcentajes de mala cobertura, mazorcas podridas y acame, la variedad V-530 de INIFAP fue la más precoz.

No obstante al comparar el H-512 y los TRC CC-343 y B-830) la diferencia es de $99 \mathrm{~kg}$, sin embargo el costo de la semilla de estos últimos vale más del $100 \%$ con respecto a la semilla del híbrido $\mathrm{H}-512$ por lo que ésta resulta mas económica.

La localidad donde obtuvieron los materiales su máxima expresión fue en el CECOT donde el rendimiento mas alto lo presentó el híbrido H-512 con $7605 \mathrm{~kg} / \mathrm{ha}$ seguido del híbrido de Cargill C-343 con 7604 kg/ha.

Por otro lado observamos que VS-536 y el híbrido Experimental de Cruza Simple HCSV-23 solo tienen un 4 y $7 \%$ menos que el promedio de los TRC con 5421 y 5235 $\mathrm{kg} / \mathrm{ha}$ respectivamente, además dichos materiales tuvieron excelentes rendimientos en cada localidad con la desventaja de que el HCSV-23 presenta una altura de planta alta, esta característica lo pone en un segundo término para las condiciones de la región centro de Veracruz, debido al acame que causan los fuertes vientos del norte.
En un tercer plano se encontraron la variedad V-530y los híbridos de Iguala D-471 x TTC-63 y D-471 x ST-549 presentando los promedios mas bajos con 4560,4370 y $3869 \mathrm{~kg} / \mathrm{ha}$ respectivamente; se aprecia en el Cuadro 2 que estos híbridas registraron los más altos porcentajes de mala cobertura y acame además de que fueron susceptibles a enfermedades en las localidades mas húmedas como Acayucan, San Sandrés Tuxtla y Papantla; lo anterior obedece a que estos materiales son de trópico seco, sin embargo son de porte bajo, tienen buena arquitectura de planta y además de ser precoces presentan buenos rendimientos motivo por el cual el programa de mejoramiento de maíz del CECOT los puede utilizar como recursos genéticos.

\section{CONCLUSIONES}

El híbrido H-512 obtuvo el rendimiento más alto a través de localidades, comportándose estable y mostró excelentes características agronómicas para el área tropical de Veracruz.

Los TRC C-343 y B-830 poseen buenos rendimientos y expresión fenotípica que los hace susceptibles de ser utilizados por el agricultor veracruzano.

La variedad sintética de maíz VS-536 tiene buena adaptabilidad en cada uno de los ambientes y obtuvo sólo 
un $4 \%$ menos de rendimiento que el promedio de los TRC.

Los híbridos de Iguala D-471 x TTC-63 y D-471 x ST-549 no se adaptaron a las condiciones tropicales de Veracruz.

\section{LITERATURA CITADA}

CALVA, J. L. 1991. Probables efectos de un tratado de libre comercio en el Campo Mexicano. Fontamara. México. p.1416.

PALAFOX, C. A. 1990. Tipificación de extensionistas y productores de Maíz en Veracruz. In: Memoria del curso teóricopráctico sobre el cultivo de maíz. PRONAMAT. Veracruz, Ver., México. p.31.
RALDA, C. G. 1979. Metodología para desarrollar un plan piloto para transferencia de tecnología en servicio. In: Memoria XXV Reunión Anual del Programa Cooperativo Centroamericano para el Mejoramiento de Cultivos Alimenticios. PCCMCA. Tegucigalpa Honduras, C.A. p. 40.

SIERRA, M. M. et al. 1985. Validación de genotipos de maíz en el estado de Veracruz. In: Informe Técnico del Programa de Maíz del CIAGOC, Veracruz, México. 17 p.

SIERRA, M. M. et al. 1993. H-512 Híbrido de Maíz de Cruza Doble para el Trópico Húmedo de México. Folleto Técnico Núm. 3. Campo Experimental Cotaxtla, Veracruz, México. $11 \mathrm{p}$. 